\title{
Efficacy of a Multicomponent Intervention for Fibromyalgia Based on Pain Neuroscience Education, Exercise Therapy, Psychological Support, and Nature Exposure (NAT-FM): Study Protocol of a Randomized Controlled Trial
}

\author{
Mayte Serrat ${ }^{1,2,3,+} \mathbb{C}^{\text {, Juan P. Sanabria-Mazo }}{ }^{2,4,5,+}{ }^{\circledR}$, Elna García-Troiteiro ${ }^{2}$, Anna Fontcuberta ${ }^{2}$, \\ Corel Mateo-Canedo ${ }^{2}$, Míriam Almirall ${ }^{1}$, Albert Feliu-Soler ${ }^{2,4,5}$ (D), \\ Jorge Luis Méndez-Ulrich ${ }^{2, *(\mathbb{D})}$, Antoni Sanz ${ }^{2, *(\mathbb{D})}$ and Juan V. Luciano ${ }^{4,5, *(\mathbb{D})}$ \\ 1 Unitat d'Expertesa en Síndromes de Sensibilització Central, Hospital de la Vall d'Hebron, 08035 Barcelona, \\ Spain; mserrat@vhebron.net (M.S.); malmirall@vhebron.net (M.A.) \\ 2 Research Group on Stress and Health, Faculty of Psychology, Universitat Autònoma de Barcelona, \\ 08193 Cerdanyola del Vallès, Spain; juanpablo.sanabria@e-campus.uab.cat (J.P.S.-M.); \\ elna.garcia@e-campus.uab.cat (E.G.-T.); anna.fontcu@gmail.com (A.F.); \\ corel.mateo@e-campus.uab.cat (C.M.-C.); a.feliu@pssjd.org (A.F.-S.) \\ 3 Escoles Universitàries Gimbernat, Universitat Autònoma de Barcelona, Sant Cugat del Vallès, \\ 08174 Barcelona, Spain \\ 4 Institut de Recerca Sant Joan de Déu, 08950 Esplugues de Llobregat, Spain \\ 5 Teaching, Research, \& Innovation Unit - Parc Sanitari Sant Joan de Déu, 08830 St. Boi de Llobregat, Spain \\ * Correspondence: jorgeluis.mendez@uab.cat (J.L.M.-U.); antonio.sanz@uab.cat (A.S.); \\ jvluciano@pssjd.org (J.V.L.); \\ Tel.: +34-93-581-44-98 (J.L.M.-U.); +34-93-581-31-36 (A.S.); +34-93-640-6350 (ext. 1-2540) (J.V.L.) \\ + These authors have contributed equally to this work and are co-first authors.
}

Received: 30 December 2019; Accepted: 14 January 2020; Published: 19 January 2020

check for updates

\begin{abstract}
The study protocol of a prospective and randomized controlled trial for the assessment of the efficacy of nature activity therapy for people with Fibromyalgia (NAT-FM) is described. The primary outcome is the mean change from baseline in the Revised Fibromyalgia Impact Questionnaire (FIQR) score at post-treatment (12 weeks) and at 9 months of follow-up, and secondary outcomes are changes in the positive affect, negative affect, pain, fatigue, self-efficacy, catastrophising, and emotional regulation. A total of 160 patients with fibromyalgia will be divided into two arms: treatment-as-usual (TAU) and NAT-FM+TAU. Pre, during, post, +6 , and +9 months assessments will be carried out, as well as an ecological momentary assessment (EMA) of intrasession and intersessions. Results will be subjected to a mixed group (NAT-FM+TAU vs. TAU) $\times$ phase (pre, post, +6 months, +9 months) general linear model. EMA intrasession measurements will be subjected to a 2 (pre vs. post) $\times 5$ (type of activity) mixed-effects ANOVA. EMA between-session measurements obtained from both arms of the study will be analysed on both a time-domain and frequency-domain basis. Effect sizes and number needed to treat (NNT) will be computed. A mediation/moderation analysis will be conducted.
\end{abstract}

Keywords: fibromyalgia; multicomponent treatment; pain neuroscience education; exercise therapy; cognitive behavioural therapy; nature exposure; randomized controlled trial; ecological momentary assessment; study protocol 


\section{Introduction}

According to the American College of Rheumatology (ACR), Fibromyalgia (FM) is a chronic disease of unknown etiology, characterised by the presence of generalized musculoskeletal pain and other symptoms, such as fatigue, waking unrefreshed, and cognitive problems [1]. The etiopathogenesis of FM involves several mechanisms, the most notable of these being the sensitization of the central nervous system (CNS). Abnormalities in the ascending and descending pathways associated with pain processing have been observed in FM [2]. The chronic nature of pain, wide range of symptoms, and comorbidity with mental disorders (mainly anxiety and depression) strongly impact on the well-being and quality of life of patients with FM [1,3,4].

Currently, the prevalence of FM among the general population is estimated to be around $2 \%$ worldwide and 2.6\% in Europe [5]. In Spain, a prevalence of 2.4\% is reported in the general population over 20 years of age, corresponding to $4.2 \%$ for women and $0.2 \%$ for men [6]. The direct costs (medical care, prescription of medicines, etc.) and indirect costs (absenteeism from work, loss of work, etc.) of FM are close to 11 billion $€$ per year [7]. Due to the variety of complex factors involved in FM, recent years have seen the development and testing of various pharmacological [8] and non-pharmacological $[9,10]$ treatments for management of the condition. The side effects associated with pharmacological treatments, along with their suboptimal clinical effects, have recently prompted the European League against Rheumatism (EULAR) to recommend that these treatments should only be used to control pain and sleep disturbances caused by FM [11].

Multicomponent treatments integrating exercise therapy (ET), cognitive behavioural therapy (CBT), and pharmacological treatment have generally been one of the most beneficial alternatives for improving quality of life, increasing functional capacity, and decreasing chronic pain in FM patients [12]. Randomized controlled trials (RCTs), testing for the simultaneous combination of ET and CBT, have been shown to be the most effective non-pharmacological treatments for FM [13]. Treatments based exclusively on ET have contributed to the reduction of symptoms of pain, fatigue, and depression, as well as to the improvement of mental health, psychological well-being, and physical function $[14,15]$. CBT-based treatments have favoured the acceptance of FM, strengthening of self-efficacy, development of coping strategies, and the reduction in depressive moods [16]. Pain neuroscience education (PNE) has been shown to be more effective than biomedical education [17] for FM and it is proving to be a key strategic piece in both the physical and cognitive approach of FM patients [18-20].

The strengths of such treatments, coupled with the identified benefits of exposure to natural contexts for people's mental health, justify the need to explore new interventions for FM patients conducted in nature. Considering the moderate benefits that have been reported for the four therapeutic components mentioned above, it seems necessary to test empirically, for the first time, the synergistic effects of its combination. There is some evidence that nature can exert a synergistic effect when added to other therapeutic ingredients [21-26]. In this context, the Nature, Activity Therapy for people with Fibromyalgia (NAT-FM) project has emerged as a new generation of therapeutic treatments for intervention of health problems, which integrates CBT, PNE [27], ET, and nature exposure. Although activity-based interventions in nature have demonstrated positive effects for treating emotional, cognitive, and behavioural functioning in both the general population [28-30] and clinical population [31,32], as far as we know there is only one clinical trial that provides empirical evidence of their efficacy in FM patients [33].

This paper describes the design characteristics of the NAT-FM treatment in comparison with a treatment-as-usual (TAU). The objectives of the RCT are the following: (a) to analyse the efficacy of the NAT-FM program as an add-on to TAU in improving the functional status (primary outcome) of FM patients; (b) to compare the efficacy of NAT-FM treatment with respect to TAU in improving affectivity, emotional regulation, perceived competence, self-esteem, self-efficacy, anxiety, depression, and other secondary outcomes; and (c) to identify factors that may act as mediators or moderators of the efficacy of NAT-FM treatment (age, years of evolution, psychological inflexibility, etc.). 


\section{Materials and Methods}

\subsection{Research Design}

The RCT protocol was developed following the Standard Protocol Items: Recommendations for Interventional Trials (SPIRIT) [34] and has been registered with ClinicalTrials.gov (Trial registration: NCT04190771). This study will employ a 9-month, randomized controlled trial (RCT) design, with two treatment arms. For the reporting of the RCT, we will follow the guidelines of the Consolidated Standards of Reporting Trials (CONSORT) [35]. The two arms of the treatment will be: (a) TAU (control group) and (b) TAU+NAT-FM (intervention group). Patients in both arms received TAU, since NAT-FM will, in principle, be a complementary treatment to that which is usually provided by the Spanish National Health System [36].

\subsection{Participants}

A total of 160 patients with FM will be recruited from the Central Sensitivity Syndromes Unit (CSSU) at the Vall d'Hebron University Hospital (Barcelona, Spain). The sample size was established using IBM SPSS SamplePower 3.0 (IBM, Chicago, IL, USA), considering the results of a meta-analysis of therapies in nature [21], which reported an average effect size of $g=0.50$, regarding clinical variables. With the anticipation of a $20 \%$ dropout and setting alpha $=0.05$ and power $1-b=0.80$, the estimated sample size is $n=80$ participants per condition. The sample size in each of the arms and the treatment follow-up period were estimated in such a way that they will be sufficiently representative to capture important results.

Patients visited consecutively by the physical therapist of the CSSU from November to December 2020 who met the selection criteria will be recruited. The inclusion criteria will be: (a) adults $\geq 18$ years old, (b) to meet the 2010-2011 ACR diagnostic criteria for FM [1,37,38], and (c) to be able to understand and agree to participate in the study. The exclusion criteria will be: (a) participating in concurrent or past RCTs (previous year) and (b) exhibiting comorbidity with severe mental disorders (e.g., psychosis) or neurodegenerative diseases (e.g., Alzheimer's) that would limit the ability of the patient to participate in the RCT.

\subsection{Procedure}

The main researcher (M.S.) will, through an initial interview, provide an overview of the study to FM patients interested in participating who met inclusion and exclusion criteria. Prior to being randomly allocated to treatments (TAU or TAU+NAT-FM), informed consent forms will be collected, which will include a detailed description of the characteristics of the interventions. Patients will also be informed that their participation will be voluntary and that they will be able to withdraw at any time, with the guarantee that they will continue to receive their usual treatment. This research will be conducted in accordance with the ethical standards set forth in the 1964 Declaration of Helsinki and was approved by the hospital's Ethics Committee (PR(AG)120/2018). Patient data will be treated confidentially, ensuring that only the research team can access this information after recoding the name and personal identity number. Only the principal investigator will have access to the patient code key, which, according to current data protection legislation in Spain, will be stored separately in a safe place. The participants will be assigned to the intervention group (TAU+NAT-FM) or control group (TAU), employing a SPSS v25 randomization list.

The pharmacological treatment will not be modified during the RCT. Only rescue paracetamol (maximum 1 gram every 8 hours) will be allowed if there is worsening of pain. Patients will be evaluated before (pre), after the 6th session of treatment (during), and after (post) treatment, as well as 6 and 9 months after baseline assessment (follow-up). Measurements shall be made according to the time established for their administration: (a) Classical structural assessment (CSA): pre, during, post, and follow-up; and (b) ecological momentary assessment (EMA): intrasession (session log) and intersessions (day log between sessions). The flow chart of the RCT is presented in Figure 1. 


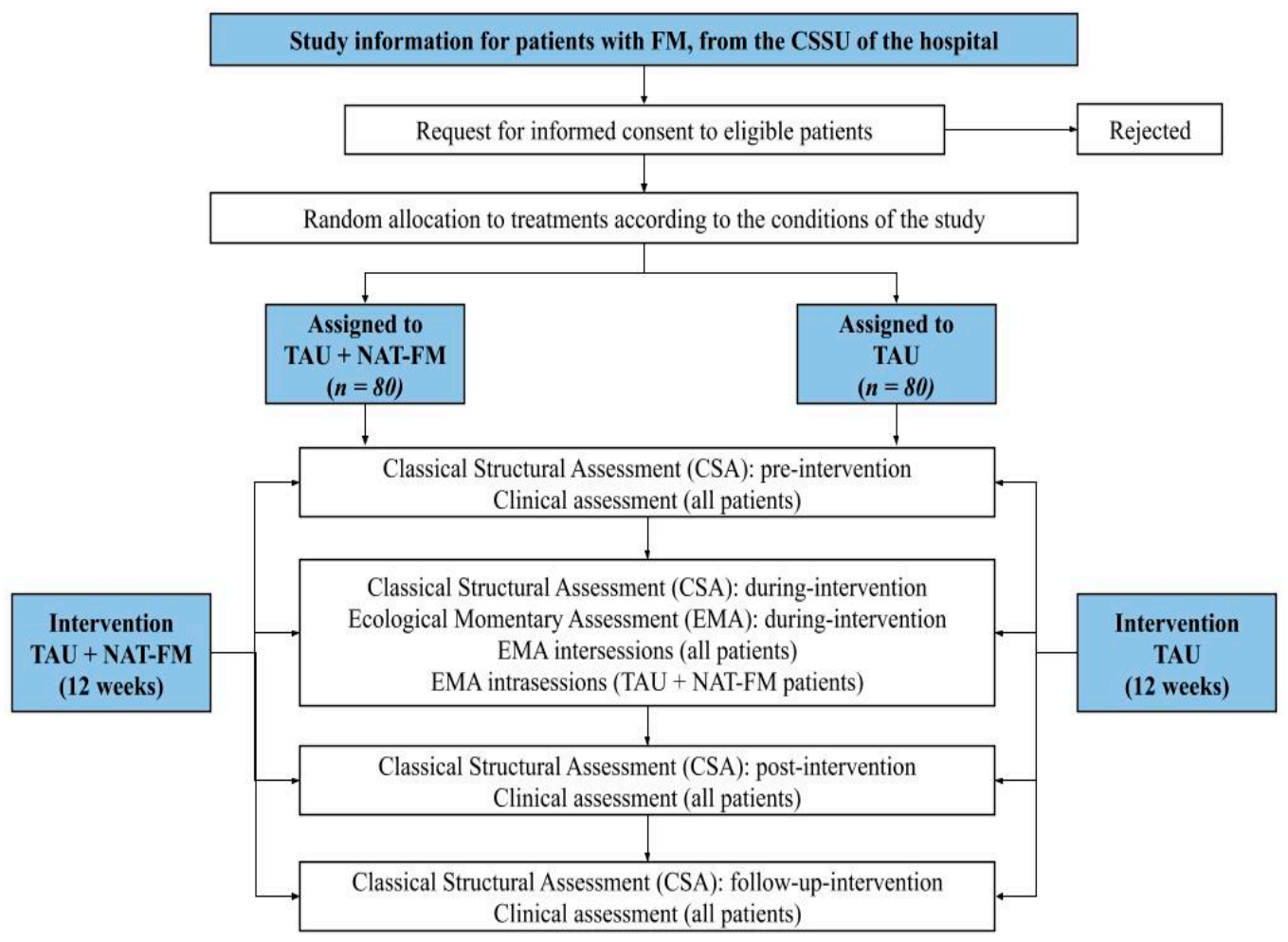

Figure 1. Flow chart of the randomized controlled trial (RCT).

Forecast Execution Dates

- Recruitment of patients: November 2019

- Finalisation of patient monitoring period: July 2020

- Publication of results: June 2021

\subsection{Treatments}

\subsubsection{Intervention Group (TAU + NAT-FM)}

NAT treatments are a new generation of therapeutic programs for health intervention, in which CBT, PNE [27], ET, and nature exposure are integrated. NAT-FM is conceived as an add-on therapy and, in particular, has the primary therapeutic objective of helping patients to improve their functional status. The secondary objectives are to contribute to improving their affectivity, emotional regulation, and self-efficacy, as well as to reduce their perception of pain, fatigue, and catastrophising. Other variables that are usually improved by these treatments are perceived competence, self-esteem, stress, sleep quality, anxiety, depression, psychological inflexibility, kinesiophobia, physical function, and functionality. Tables 1 and 2 show an outline of the NAT-FM.

The NAT-FM design process was guided by the procedures established in the protocol and the proof of concept study. The therapeutic objectives were selected by taking into account the results of a systematic review of the psychological characteristics of people with FM (affective, cognitive, metacognitive, and personality profiles) [39] and the validation of a panel of experts made up of researchers and psychologists (M.S., J.P.S.-M., A.F.-S., J.L.M.-U., J.V.L, and A.S.), who evaluated the existing scientific evidence and the clinical and investigative relevance of each objective. The panel concluded that the processes to be included in the treatment are self-efficacy, negative affect, positive affect, emotional regulation, and catastrophising. The activities indicated in Table 1 were selected considering the results of an empirical study on the therapeutic potential of 10 activities in nature [40]. The number of activities practiced in each session was adapted according to the established targets, taking into account the progression of the patients and the evolution of the treatment. The 
activities addressing each target were selected considering the therapeutic potential identified by the expert panel.

The sectors (natural spaces) in which the outdoor sessions will be conducted have been validated by experts to guarantee their suitability for the treatment activities [40]. The geographical areas are Sant Genís Forest (coordinates N $41^{\circ} 25.930$ E $2^{\circ}$ 8.237) and Les Escletxes del Papiol (coordinates N $41^{\circ} 26.319 \mathrm{E} 2^{\circ} 1.101$ ). The researchers conducted a proof of concept study (J.P.S.-M., E.G.-T., C.M.-C., and A.S) in these sectors to test the manualisation of procedures and to evaluate the suitability of the NAT-FM structure based on the testing of a series of representative sessions [41].

The protocol of the NAT-FM is the result of the consensus of the proposed instruments, of the moments of assessments corresponding to each phase of the treatment, and of the administration platforms used. The decision to combine different assessments (CSA+EMA) is motivated by the need for a strategy that could obtain more precise information about the dynamics of the variables to be evaluated and, in particular, could record the affective and cognitive impact of each activity, as well as its transfer to everyday life. The frequency of administration of the EMA has been established from analysing the results of a systematic review on the characteristics of its use in studies with patients with chronic pain [42].

The NAT-FM treatment will be carried out in a group-based format in groups made up of a maximum of 20 patients per session with a total of 12 weekly $2 \mathrm{~h}$ sessions ( 1 session per week). The sessions will be directed by the M.S., who is a physiotherapist of the CSSU of Vall d'Hebron University Hospital and also a psychologist and sports technician with the required legal qualifications for conducting this activity.

\subsubsection{Control Group (TAU)}

The TAU provided to patients in the control group of this study is based primarily on the prescription of drugs adjusted to the symptomatic profile of each patient, with complementary advice on aerobic exercise and pain neuroscience education adapted to the physical capacities of the patients. Patients of the control group will be placed on a waiting list so that at the end of the RCT they can benefit from the NAT-FM treatment.

Table 1. Structure of the Nature Activity Therapy for people with Fibromyalgia (NAT-FM), indicating the activities to be performed and the psychological targets to be addressed in each of the sessions.

\begin{tabular}{|c|c|c|c|c|c|c|c|c|c|c|c|}
\hline \multirow{2}{*}{ Session } & \multicolumn{5}{|c|}{ Activities } & \multicolumn{6}{|c|}{ Targets } \\
\hline & NW & YO & HK * & PH & SY** & $\begin{array}{l}\text { Negative } \\
\text { Affect }\end{array}$ & $\begin{array}{c}\text { Positive } \\
\text { Affect }\end{array}$ & Pain/Fatigue & $\begin{array}{l}\text { Emotional } \\
\text { Regulation }\end{array}$ & Self-Efficacy & Catastrophising \\
\hline 1 & & & $x$ & & & & & $1-16$ & & & \\
\hline 2 & & $x$ & $x$ & & & & $1-6$ & $1-2$ & & & \\
\hline 3 & & $x$ & $x$ & $x$ & & & & $3-4$ & & $1-6$ & \\
\hline 4 & $x$ & & $x$ & & & & & $5-6$ & & $7-10$ & \\
\hline 6 & $x$ & $x$ & $x$ & & & $1-6$ & & $9-10$ & & & \\
\hline 7 & $x$ & & $x$ & & $x$ & & & $11-12$ & & & $1-6$ \\
\hline 8 & $x$ & & $x$ & $x$ & & & & $13-14$ & $1-5$ & & \\
\hline 9 & & $x$ & $x$ & & & & & $15-16$ & $6-8$ & & \\
\hline 10 & & & $x$ & $x$ & & \multicolumn{6}{|c|}{ Family session } \\
\hline
\end{tabular}

Note. The " $X$ " expresses the sessions in which each activity is planned. The numbers of the targets' columns are referred to the steps described on Table 2. Cognitive behavioural intervention on primary and secondary outcomes is distributed along the sessions. ${ }^{*}$ Hiking is a homework assignment to do as a therapeutic exercise with cognitive targets. ${ }^{* *}$ Shinrin Yoku is understood as mindfulness in a natural context. Nordic walking (NW); yoga (YO); hiking $(\mathrm{HK})$; photography (PH); Shinrin Yoku (SY). 
Table 2. Steps in pain neuroscience education and in the cognitive-behavioural treatment for each outcome.

\begin{tabular}{|c|c|}
\hline \multicolumn{2}{|r|}{ Pain/Fatigue (Pain Neuroscience Education) } \\
\hline 1 & Disassembling beliefs. \\
\hline 2 & Concept of central nervous system and centre sensitization. \\
\hline 3 & Concept of pain, fatigue, and pain system. \\
\hline 4 & Acute pain vs chronic pain: Purpose of acute pain and how it originates in the nervous system. \\
\hline 5 & Pain and damage. \\
\hline 6 & Pain neuromatrix theory and virtual body representation. \\
\hline 7 & Nociception, nociceptors, action potential, peripheral sensitization, synapses. \\
\hline 8 & Descending/ascending pain pathways, spinal cord. \\
\hline 9 & Danger signs: modulation and modification. \\
\hline 10 & Hyperalgesia and allodynia, hypersensitivity of nervous central system. \\
\hline 11 & The role of the brain, pain memory, pain perception, autoimmune evaluation error. \\
\hline 12 & Etiology. \\
\hline 13 & Relationship with stress and emotions. \\
\hline 14 & Relationship with attention, perceptions, pain cognitions, and pain behaviour. \\
\hline 15 & Neuroplasticity and how pain becomes chronic. \\
\hline 16 & Re-education, graded activity, and exercise therapy \\
\hline \multicolumn{2}{|r|}{ Self-Efficacy } \\
\hline 1 & Understand the concept of self-efficacy. \\
\hline 2 & Recognize the relationship between self-efficacy, pain, and fatigue. \\
\hline 3 & Recognize the importance of adapting self-efficacy to real capacity. \\
\hline 4 & Become aware of the process of self-efficacy elaboration. \\
\hline 5 & Recognize the dynamics of self-efficacy. \\
\hline 6 & Identify the sources of self-efficacy. \\
\hline 7 & Identify the biases in the creation of self-efficacy. \\
\hline 8 & Learn how to stop and replace the biases in the creation of self-efficacy. \\
\hline 9 & Relationship between self-efficacy and self-esteem. \\
\hline 10 & Transfer: How to work self-efficacy in day life. \\
\hline \multicolumn{2}{|r|}{ Positive Affect } \\
\hline 1 & Understand the concept of positive emotions. \\
\hline 2 & Recognize the relationship between positive emotions, pain, and fatigue. \\
\hline 3 & Recognize the importance of actively searching for sources of positive emotions. \\
\hline 4 & Identify sources of positive emotions in the context of the sessions. \\
\hline 5 & Learn to pay attention to stimuli/conditions that generate positive emotions. \\
\hline 6 & Transfer: How to work positive affect in everyday life. \\
\hline \multicolumn{2}{|r|}{ Negative Affect } \\
\hline 1 & Understand the concept of negative emotions. \\
\hline 2 & Recognize the relationship between negative emotions, pain, and fatigue. \\
\hline 3 & Recognize the adaptive function of negative emotions. \\
\hline 4 & Assess the importance of emotional regulation to reduce negative emotions. \\
\hline 5 & Learn to pay attention to the stimuli/conditions that generate negative emotions. \\
\hline 6 & Transfer: How to reduce negative affect to everyday life. \\
\hline \multicolumn{2}{|r|}{ Emotional Regulation } \\
\hline 1 & Understand the concept of emotional regulation. \\
\hline 2 & Recognize the relationship between emotional regulation, pain, and fatigue. \\
\hline 3 & Identify the 9 types of cognitive regulation of emotions. \\
\hline 4 & Identify the relationship between emotional regulation, pain, and fatigue. \\
\hline 5 & Learn to pay attention to emotional regulation. \\
\hline 6 & Learn to identify the type of emotional regulation usually employed in everyday life. \\
\hline 7 & Learn to stop and subtract inappropriate emotional regulation for a proper one. \\
\hline 8 & Transfer: How to work emotional regulation in everyday life. \\
\hline \multicolumn{2}{|r|}{ Catastrophising } \\
\hline 1 & Understand the concept of catastrophism. \\
\hline 2 & Recognize the relationship between catastrophism, pain, and fatigue. \\
\hline 3 & Learn to recognize the catastrophic thoughts. \\
\hline 4 & Learn to pay attention to the catastrophic thoughts. \\
\hline 5 & Learn to stop and replace the catastrophic thoughts. \\
\hline 6 & Transfer: How to work catastrophising on an everyday basis. \\
\hline
\end{tabular}

Note. To present in a most understandable way this information to patients, a power point has been used with pictures, examples, and metaphors, according to the recommendations [18]. All these aspects have been reinforced point by point in each session with the book Explain Pain in Spanish [27]. 


\subsection{Study Measures}

The participants of the intervention group (TAU+NAT-FM) and the control group (TAU) will complete the instruments described below. Assessment will be organized considering the timings (CSA+EMA) that were established from the results of the proof of concept study [41]. Table 3 shows the measurement scheme and timings for the NAT-FM treatment protocol.

Table 3. Measurement scheme and timings for the NAT-FM treatment protocol.

\begin{tabular}{|c|c|c|c|c|c|}
\hline & Pre & $\begin{array}{l}\text { During } \\
\text { (Session 6) }\end{array}$ & Post & $\begin{array}{l}\text { Follow-Up } \\
\text { (6 Months) }\end{array}$ & $\begin{array}{l}\text { Follow-Up } \\
\text { (9 Months) }\end{array}$ \\
\hline \multicolumn{6}{|c|}{ Classical Structural Assessment (CSA) } \\
\hline \multicolumn{6}{|l|}{ General information measures } \\
\hline $\begin{array}{l}\text { Sociodemographic information (age, } \\
\text { education level, etc.) }\end{array}$ & $x$ & & & & \\
\hline $\begin{array}{l}\text { Clinical information (medical history, FM } \\
\text { history, etc.) }\end{array}$ & $\mathrm{x}$ & & & & \\
\hline \multicolumn{6}{|l|}{ Measures of clinical features and screening } \\
\hline SCID-I (depression module) & $x$ & & & & \\
\hline \multicolumn{6}{|l|}{ Measures of primary outcome } \\
\hline FIQR (functional status) & $\mathrm{X}$ & $X$ & $X$ & $X$ & $X$ \\
\hline \multicolumn{6}{|l|}{ Measures of secondary outcomes } \\
\hline PANAS (negative and positive affect) & $x$ & $x$ & $x$ & $x$ & $x$ \\
\hline PSS-4 (stress) & $x$ & $x$ & $x$ & $x$ & $x$ \\
\hline \multicolumn{6}{|l|}{ Measures of additional secondary outcomes } \\
\hline HADS (anxiety and depression) & $x$ & $x$ & $x$ & $x$ & $x$ \\
\hline PIPS (psychological inflexibility) & $x$ & $x$ & $x$ & $x$ & $x$ \\
\hline TSK-11 (kinesiophobia) & $x$ & $x$ & $x$ & $x$ & $x$ \\
\hline SF-36 (physical function) & $x$ & $x$ & $x$ & $x$ & $x$ \\
\hline UKK (functionality) & $x$ & $x$ & $x$ & $x$ & $x$ \\
\hline AEAC (adverse effects) * & & & $x$ & & \\
\hline PGIC/PSIC (impression of change) * & & & $x$ & & \\
\hline \multicolumn{6}{|c|}{ Ecological Momentary Assessment (EMA) } \\
\hline
\end{tabular}

Note: " $\mathrm{X}$ " expresses the moment when each assessment instrument is planned to be administered. Structured Clinical Interview for DSM Axis I Disorders (SCID-I); Revised Fibromyalgia Impact Questionnaire (FIQR); Positive and Negative Affect Schedule (PANAS); Cognitive Emotion Regulation Questionnaire (CERQ); Personal Perceived Competence Scale (PPCS); Rosenberg Self-Esteem Scale (RSES); Pain Catastrophizing Scale (PCS); Perceived Stress Scale (PSS); Hospital Anxiety and Depression Scale (HADS); Psychological Inflexibility in Pain Scale (PIPS); Tampa Scale for Kinesiophobia (TSK-11); 36-Item Short Form Survey (SF-36); UKK Walk Test (UKK); Adverse Effects Assessment Checklist (AEAC); Patient Global Impression of Change (PGIC); Pain Specific Impression of Change (PSIC); Self-Assessment Manikin (SAM); Visual Analog Scale (VAS). * Only for NAT-FM arm.

\subsubsection{Classical Structural Assessment (CSA)}

The CSA will measure general information, clinical characteristics and screening, primary outcomes, secondary outcomes, and additional secondary outcomes. These instruments will be applied to patients in both the control group (TAU) and intervention group (TAU+NAT-FM).

\section{General Information Measures}

The Socio-demographic and clinical questionnaire will be used to obtain general and clinical patient data (age, educational level, socioeconomic status, marital status, ethnic group, personal medical history, years of FM diagnosis, comorbid medical conditions, etc.). 
Measures of Clinical Features and Screening

The Structured Clinical Interview for DSM Axis I Disorders (SCID-I) [43] will be used for the diagnosis of mood disorders. It is based on the research version of SCID-I and the DSM-IV criteria.

\section{Primary Outcome Measure}

The Revised Fibromyalgia Impact Questionnaire (FIQR) comprises three dimensions: physical dysfunction (scores from 0 to 30), overall impact (scores from 0 to 20), and intensity of the symptoms (scores from 0 to 50), which are used to measure the impact generated by FM during the last week. It consists of 21 items, which are answered on a numerical rating scale of 11 points (from 0 to 10). Total scores can range from 0 to 100, with higher scores reflecting greater deterioration. The Spanish version has an adequate internal consistency $(\alpha=0.93)$ and acceptable test-retest reliability $(r=0.84)$ [44].

\section{Secondary Outcome Measures}

The Positive and Negative Affect Schedule (PANAS) is used to evaluate positive and negative affects. It consists of two dimensions (positive affect and negative affect) of 10 items, each answered on a 5-point Likert scale. Total scores of each scale range from 10 to 50, where higher scores indicate a greater presence of the specific affectivity. The Spanish version has an adequate internal consistency for the positive affect $(\alpha=0.92)$ and for the negative affect $(\alpha=0.88)$ [45].

The Cognitive Emotion Regulation Questionnaire (CERQ) is used to assess individual differences in the cognitive regulation of emotions. The instrument measures nine 2-item dimensions (self-blame, blaming others, acceptance, refocusing on planning, positive refocusing, rumination, positive reappraisal, putting into perspective, and catastrophising). This study will use the short 18-item version. Responses are given on a 5-point Likert scale ranging from 1 (almost never) to 5 (almost always). Total scores for each dimension range from 2 to 10, with the highest scores indicating the specific cognitive strategy most used. The Spanish version has an adequate internal consistency $(\alpha=$ 0.77 to 0.93$)$ and acceptable test-retest reliability ( $r=0.60$ to 0.85$)$ for the subscales [46].

The Personal Perceived Competence Scale (PPCS) is used to measure perceived competence. It consists of eight items that are answered on a 6-point Likert scale. Total scores of each scale rangefrom 8 to 48 , with higher scores indicating greater perceived competence. The Spanish version has an adequate internal consistency $(\alpha=0.83)$ [47].

The Rosenberg Self-Esteem Scale (RSES) is used to measure self-esteem. It consists of 10 items that are answered on a 4-point Likert scale. Total scores of each scale range from 10 to 40, where higher scores indicate higher self-esteem. The Spanish version has an adequate internal consistency $(\alpha=0.87)$ and acceptable test-retest reliability ( $r=0.72$ to 0.74$)$ [48].

The Pain Catastrophizing Scale (PCS) is used to evaluate catastrophic thoughts associated with pain. It consists of three dimensions (rumination, magnification, and helplessness) of 13 items in total, which are answered on a 5-point Likert scale. Total scores on each scale range from 0 to 52, with higher scores indicating more catastrophising. The Spanish version has an adequate internal consistency $(\alpha=$ 0.79) and acceptable test-retest reliability $(r=0.84)$ [49].

The Perceived Stress Scale (PSS) is used to evaluate the stress perceived by people during the previous month. This study will use a 4-item version with a 5-point Likert type response format. Total scores range from 0 to 16 , with higher scores indicating greater perceived stress. The Spanish version has an acceptable internal consistency $(\alpha=0.77)$ [50].

\section{Additional Secondary Outcomes Measures}

The Hospital Anxiety and Depression Scale (HADS) is used to quantify the severity of anxiety and depression symptoms. It consists of two dimensions (anxiety and depression) of seven items, each responding on a 4-point Likert scale. Total scores of each scale (HADS-A and HADS-D) range from 0 
to 21 , where higher scores indicate greater severity of symptoms. The Spanish version has an adequate internal consistency for anxiety ( $\alpha=0.83)$ and for depression $(\alpha=0.87)$ [51,52].

The Psychological Inflexibility in Pain Scale (PIPS) is used to assess psychological inflexibility in patients with pain. It consists of two dimensions (avoidance and cognitive fusion with pain) of 12 items, which are answered on a 7-point Likert scale. Total scores of each scale range from 12 to 84 , with higher scores indicating greater psychological inflexibility. The Spanish version has an adequate internal consistency $(\alpha=0.90)$ and test-retest reliability $(r=0.97)$ [53].

The Tampa Scale for Kinesiophobia (TSK-11) is used to assess fear of pain and movement. It consists of 11 items, which are answered on a 4-point Likert scale. Total scores of each scale range from 11 to 44 , where higher scores indicate a greater fear of pain and movement. The Spanish version has an adequate internal consistency $(\alpha=0.79)$ [54].

The physical function of the 36-Item Short Form Survey (SF-36) will be used to assess the physical function typically affected in patients with chronic pain. This dimension comprises a total of 10 items, which are answered on a Likert scale of 3 points. Total scores on each scale range from 0 to 100, with higher scores indicating better physical function. The dimensions have shown optimal internal consistency $(\alpha=0.94)$ [55].

The UKK Walk Test (UKK) [56] is used to assess people's physical fitness, endurance, and cardio-respiratory capacity (maximum $\mathrm{VO}^{2}$ ). The test is performed in a straight line with no difference in height between the two extremes. In this study, the distance from the beginning to the end of the route will be adapted from $2 \mathrm{~km}$ (original version) to $1 \mathrm{~km}$, given the specific characteristics of the population of this study. In addition, we will use Polar Advantage heart rate monitors with their respective software to mark the times and obtain the average heart rate of the patients during the route.

The Adverse effects Assessment Checklist [57] is an ad hoc measure to check for potential adverse events (e.g., headaches, dizziness, physical injuries) across the interventions and follow-up.

The Patient Global Impression of Change (PGIC) [58] and Pain Specific Impression of Change (PSIC) [57] are self-reported measures frequently used as indicators of meaningful change in treatments for chronic pain. Responses are given on a 7-point Likert scale ranging from 1 (much better) to 5 (much worse). The PGIC is one item that refers to the perception of global improvement, whereas the PSIC asks about the impression of change in more specific domains (physical and social functioning, work-related activities, mood, and pain). These scales will be completed by the participants assigned to the treatment (NAT-FM+TAU) group.

\subsubsection{Ecological Momentary Assessment (EMA)}

EMA is used to assess: (a) the specific short-term impact of each activity and (b) the transfer of the treatment effects to everyday life. Measurements during the sessions (intrasession assessment) will be taken before and after each activity in nature using an online form. Measurements of the daily records between sessions (intersession assessment) will be carried out through an app, in which patients will have to respond six times a day (twice in the morning, twice in the afternoon, and twice at night). Intersession instruments will be applied to patients in both the control group (TAU) and intervention group (TAU+NAT-FM). However, intrasession instruments will only be applied to patients in the intervention group (TAU+NAT-FM).

\section{Other Secondary Outcome Measurements}

The Self-Assessment Manikin (SAM) [59] is used to assess the affective state of people. It consists of three blocks of diagrammed pictograms in a continuous line, representing the following three dimensions of the affective response: valence, arousal, and dominance. Each scale has a 9-point Likert response format. The total scores on each scale range from 1 to 9 . Higher scores on valence indicate greater positive mood; higher scores on arousal indicate greater activation and alertness; whilst for dominance a higher score indicates greater perception of control and personal confidence. These 
three dimensions of the affective response were evaluated both in intrasession assessment and in intersession assessment.

Single-item questionnaires: For the assessments of the variables fatigue, pain, and sleep quality, the three items of the Visual Analog Scale (VAS) of the FIQR were selected. For stress and self-efficacy, ad hoc questions were designed with a single item, rated on a scale from 0 to 10 , in a VAS format. Higher scores indicate greater perceived fatigue, pain, sleep quality, stress, and self-efficacy. The variables fatigue, pain, and sleep quality were evaluated by intersession assessment, whereas the variables energy, pain, stress, and self-efficacy were evaluated by intrasession assessment.

\subsection{Statistical Analysis}

Data analyses will be conducted by means of the statistical program SPSS v25 and MPlus 7.0. Initially, descriptive statistics will be calculated for all measures of the study (general, clinical characteristics/screening, and primary/secondary/additional secondary outcomes). Subsequently, multivariate analyses will be conducted to define the effect size of the intervention at the different assessment periods: CSA (pre, during, post, and follow-up) and EMA (during). Continuous variables will be analysed using the Kolmogorov-Smirnov test to assess normal distribution and the Levene test for equal variances. First, we will use the Student's t-test to examine the baseline between-group differences in sociodemographic and clinical characteristics. The primary between-group analysis to assess the effect of treatment will be conducted on an intention-to-treat (ITT) basis with the FIQR total score as a continuous variable and assuming data missing at random. This involves using 2 $\times 4$ mixed-effects ANOVA with the group (TAU+NAT-FM vs. TAU) as the between-groups factor and phase (pre, post, follow-up +6 , follow-up +9 ) as the within-subjects factor. Corrected $\eta p^{2}$ (partial eta-square) will be estimated for the full model (group and phase main effects and group $x$ phase interaction). We will also report the effect size (Cohen's $d$ ) for each pairwise comparison, using the pooled baseline SD to weight the differences in the pre-post means and to correct for the population estimate. Separate models will be estimated for each of the secondary outcomes using the same analytical strategy.

In addition, to assess the clinical significance of the improvement in the primary outcome (FIQR), we will classify participants into two categories (responders vs. non-responders to treatment), using the following criterion: $\geq 20 \%$ reduction in the pre-post FIQR total scores [60]. This classification will be used to compute the number needed to treat (NNT) in NAT-FM compared with TAU. Finally, we will examine whether the effect of NAT-FM on the primary outcome at post and at the 6 and 9-month follow-ups is mediated/moderated through pre-post changes in secondary measures and personal factor variables (age, years from diagnosis, severity), using a structural equation model. According to the proposal of Luciano et al. [60], change scores before and after NAT-FM treatment will be calculated for these variables, as well as change scores before follow-up for the outcome variables. The analysis of temporality will increase the possibility of drawing conclusions about causality relationships. In this process, the data of the patients of the treatment with the greatest participation in the intervention will be analysed, defining participation as a minimum of $75 \%$ attendance of the sessions (per protocol analysis). The Benjamini-Hochberg correction for multiple comparisons will be applied; a procedure to detect false findings designed to overcome the limitations of other common procedures [61].

Complementary analyses will be conducted. Firstly, EMA intrasession measurements obtained from the NAT-FM treatment group will be subjected to a 2 (pre vs. post) $\times 6$ (type of activity) mixed-effects ANOVA for pain, fatigue, self-efficacy, stress, and affect in order to assess the short-term impact of the planned activities. Secondly, EMA between-session measurements obtained from both arms of the study will be analysed both on a time-domain (single regression linear model for each dependent variable) and a frequency-domain basis (spectral analysis for each dependent variable) in order to assess transfer of the treatment effects to daily life. 


\section{Discussion}

The main objective of this article is to report the design characteristics of the NAT-FM treatment in comparison with TAU. The protocol for this RCT was designed using SPIRIT recommendations and recorded in a clinical studies database (ClinicalTrials.gov) in accordance with CONSORT guidelines. If the findings of the RCT are sufficiently robust, NAT-FM treatment could be offered as an add-on intervention to the usual treatment (TAU), with a coherent strategic approach that is adjustable to the health resources allocated to FM patients. In this RCT, in addition to evaluating the clinical effects of NAT-FM treatment in the mid- and long-term, we will seek to recognize relevant moderators and mediators of clinical change.

The possible withdrawal of patients from the trial will be one of the risk components that will be considered. In this regard, two strategies will be developed: (a) conduct a sensitivity analysis to determine the impact of adherence to the NAT-FM protocol on the observed effects and (b) combine this RCT to the MOTI-NAT programme (Motivational intervention for Nature Activity Therapy) [62], which will have as its main objective the development of specific therapeutic adherence strategies for patients linked to the different protocols of the NAT Project based on the increased motivation to participate in these therapies.

NAT-FM treatment is presented as the first intervention that integrates CBT, PNE [27], ET, and nature exposure. The integrative commitment of this RCT is based on the recognition of the scientific evidence identified in different studies in the components of treatment. The results obtained in the proof of concept study [41], designed as a preparation for this protocol, suggest moderate to strong size effects of the therapy, which constitutes an empirical basis for the rationale underlying the intervention.

The results derived from the planned full trial are expected to constitute a preliminary step towards the development of this new generation of therapeutic treatments for intervention in various health problems based on CBT combined with PNE and ET in natural contexts. A deeper understanding of the specific therapeutic effects of NAT-FM could be very informative when considering the relevance of this approach as a complementary model of health intervention, specifically in the domain of chronic pain. With regards to FM, it is worth remembering that there are currently no curative treatments and that developing new approaches that improve the functionality of patients would be of great benefit both at a social and personal level (due to the high impact and prevalence of this condition), particularly given the healthcare (high use of public resources) and economic (high consumption of health resources/job losses) consequences of this illness.

\section{Conclusions}

This study represents the first attempt for combining pain neuroscience education, exercise therapy, psychological therapy, and exposure to nature in the treatment of fibromyalgia.

- A randomized controlled two-arm trial is planned to address safety and potential efficacy of NAT-FM (Nature Activity Therapy for Fibromyalgia) in comparison with traditional care (TAU).

- NAF-FM combines the classical structural assessment (CSA) and ecological momentary assessment (EMA) to obtain more precise information about the dynamics of the variables to be evaluated, to record the affective and cognitive impact of each activity, and to identify its transfer to everyday life.

- As indicated in previous studies, there is scientific evidence that the improvement in functional status of the people diagnosed with fibromyalgia can be attained by the direct intervention in processes such as the positive and negative affect, self-efficacy, pain, fatigue, emotional regulation, and catastrophising.

- Some issues arise from the complexity of combining the components of the intervention, particularly those related to logistics and the diversity of activities to be completed by the participants, and the determining the 'effective' treatment components. 
Author Contributions: Systematic revision of the literature and conceptualization, M.S., J.P.S.-M., J.L.M.-U. and A.S.; panel of experts for the analysis of the therapeutic potential, M.S., J.L.M.-U. and A.S.; MOTI-NAT-FM study for the analysis of the determinants for initiation and adherence to the programme, M.S., J.P.S.-M., C.M.-C., J.L.M.-U. and A.S.; treatment design, M.S., J.P.S.-M., M.A., A.F.-S., J.L.M.-U., J.V.L. and A.S.; protocol design, M.S., J.P.S.-M., E.G.-T., A.F., M.A., A.F.-S., J.V.L. and A.S.; instruments selection, M.S., J.P.S.-M., M.A., A.F.-S., J.V.L. and A.S.; proof-of-concept, J.P.S.-M., E.G,-T., C.M.-C. and A.S.; paper writing, reviewing, and editing, M.S., J.P.S.-M. and A.S.; paper revision, M.S., J.P.S.-M., C.M.-C., M.A., A.F.-S., J.L.M.-U., J.V.L. and A.S.; original draft preparation, J.P.S.-M.; ethical approval and trial registration, M.S., M.A. and J.V.L. All authors have read and agree to the published version of the manuscript.

Funding: This research was funded by the Vall d'Hebron Institute of Research Funding, Autonomous University of Barcelona (Ideas Generation Program, Price 2018), and Parc Sanitari Sant Joan de Déu. A.F.-S. has a Sara Borrell contract and J.V.L has a Miguel Servet contract, awarded both by the Institute of Health Carlos III (ISCIII; CD16/00147 and CPII19/00003, respectively). The funding bodies did not have any role in the collection, analysis, and interpretation of data; in the writing of the report; and in the decision to submit the article for publication.

Conflicts of Interest: The authors declare no conflict of interest.

\section{References}

1. Wolfe, F.; Clauw, D.J.; FitzCharles, M.; Goldenberg, D.L.; Häuser, W.; Katz, R.L.; Mease, P.J.; Russell, A.S.; Russell, I.J.; Walitt, B. Revisions to the 2010/2011 Fibromyalgia diagnostic criteria. Arthritis Rheum. 2016, 46, 319-329. [CrossRef] [PubMed]

2. Häuser, W.; Ablin, J.; Fitzcharles, M.A.; Littlejohn, G.; Luciano, J.V.; Usui, C.; Walitt, B. Fibromyalgia. Nat. Rev. Dis. 2015, 1, 15022. [CrossRef] [PubMed]

3. Sluka, K.A.; Clauw, D.J. Neurobiology of fibromyalgia and chronic widespread pain. Neuroscience 2016, 3, 114-129. [CrossRef]

4. Sanabria-Mazo, J.P.; Gers-Estrada, M. Implications of chronic pain on the quality of life of women with fibromyalgia. Psicol. Estud. 2018, 23, 81-89. [CrossRef]

5. Heidari, F.; Afshari, M.; Moosazedeh, M. Prevalence of fibromyalgia in general population and patients, a systematic review and meta-analysis. Rheumatol. Int. 2017, 37, 1527-1539. [CrossRef] [PubMed]

6. Mas, A.J.; Carmona, L.; Valverde, M.; Ribas, B.; EPISER Study Group. Prevalence and impact of fibromyalgia on function and quality of life in individuals from the general population: Results from a nationwide study in Spain. Clin. Exp. Rheumatol. 2008, 26, 519-526.

7. Rivera, J.; Rejas, J.; Esteve, J.; Vallejo, M. Costes Económicos Asociados al Diagnóstico de Fibromialgia en Spain; Congreso EULAR: Copenhague, Dinamarca, 2009.

8. Häuser, W.; Ablin, J.; Perrot, S.; Fitzcharles, M.A. Management of fibromyalgia: Practical guides from recent evidence-based guidelines. Pol. Arch. Med. Wewn. 2017, 127, 47-56. [CrossRef]

9. Feliu-Soler, A.; Borràs, X.; Peñarrubia-María, M.T.; Rozadilla-Sacanell, A.; D'Amico, F.; Moss-Morris, R.; Howard, M.A.; Fayed, N.; Soriano-Mas, C.; Puebla-Guedea, M.; et al. Cost-utility and biological underpinnings of Mindfulness-Based Stress Reduction (MBSR) versus a psychoeducational programme (FibroQoL) for fibromyalgia: A 12-month randomised controlled trial (EUDAIMON study). BMC Complement. Altern. Med. 2016, 16, 81. [CrossRef]

10. Haugmark, T.; Hagen, K.B.; Provan, S.A.; Bærheim, E.; Zangi, H.A. Effects of a community based Multicomponent rehabilitation programme for patients with fibromyalgia: Protocol for a randomized controlled trial. BMJ Open 2018, 8, e021004. [CrossRef]

11. Macfarlane, G.J.; Kronisch, C.; Dean, L.E.; Atzeni, F.; Häuser, W.; Fluß, E.; Choy, E.; Kosek, E.; Amris, K.; Branco, J.; et al. EULAR revised recommendations for the management of fibromyalgia. Ann. Rheum. Dis. 2017, 76, 318-328. [CrossRef]

12. Häuser, W.; Bernardy, K.; Arnold, B.; Offenbächer, M.; Schiltenwolf, M. Efficacy of multicomponent treatment in fibromyalgia syndrome: A meta-analysis of randomized controlled clinical trials. Arthritis Rheum. 2009, 61, 216-224. [CrossRef] [PubMed]

13. Nishishinya, M.B.; Rivera, J.; Alegre, C.; Pereda, C.A. Non-pharmacologic and alternative treatments in fibromyalgia. Med. Clin. 2016, 127, 295-299. [CrossRef]

14. Nelson, N.L. Muscle strengthening activities and fibromyalgia: A review of pain and strength outcomes. J. Bodyw. Mov. Ther. 2015, 19, 370-376. [CrossRef] [PubMed] 
15. Sosa, M.D.; Núñez, S.; Gallego, T.; Pecos, D.; Monserrat, J.; Álvarez, M. Effectiveness of therapeutic exercise in fibromyalgia syndrome: A systematic review and meta-analysis of randomized clinical trials. Biomed. Res. Int. 2017. [CrossRef]

16. Bernardy, K.; Klose, P.; Welsch, P.; Häuser, W. Efficacy, acceptability, and safety of cognitive behavioural therapies in fibromyalgia syndrome: A systematic review and meta-analysis of randomized controlled trials. Eur. J. Pain 2018, 22, 242-260. [CrossRef] [PubMed]

17. Amer-Cuenca, J.J.A.; Pecos-Martín, D.; Martínez-Merinero, P.; Lluch, E.; Nijs, J.; Meeus, M.; Peña, R.F.; Fernández-Carnero, J. How much is needed? Comparison of the effectiveness of different pain education dosages in patients with fibromyalgia. Pain Med. 2019, 1-12. [CrossRef]

18. Nijs, J.; Paul van Wilgen, C.; Van Oosterwijck, J.; van Ittersum, M.; Meeus, M. How to explain central sensitization to patients with 'unexplained' chronic musculoskeletal pain: Practice guidelines. Man. Ther. 2011, 16, 413-418. [CrossRef]

19. Moseley, G.L. Evidence for a direct relationship between cognitive and physical change during an education intervention in people with chronic low back pain. Eur. J. Pain 2004, 8, 39-45. [CrossRef]

20. Van Oosterwijck, J.; Meeus, M.; Paul, L.; De Schryver, M.; Pascal, A.; Lambrecht, L.; Nijs, J. Pain physiology education improves health status and endogenous pain inhibition in fibromyalgia: A double-blind randomized controlled trial. Clin. J. Pain 2013, 29, 873-882. [CrossRef]

21. Bowen, D.J.; Neill, J.T. A meta-analysis of adventure therapy outcomes and moderators. Open Psychol. J. 2013, 6, 28-53. [CrossRef]

22. Bowen, D.J.; Neill, J.T.; Crisp, S.J. Wilderness adventure therapy effects on the mental health of youth participants. Eval. Program. Plann. 2016, 58, 49-59. [CrossRef] [PubMed]

23. Cole, H.; Triguero, M.; Connolly, J.; Anguelovski, I. Determining the health benefits of green space: Does gentrification matter? Health Place 2019, 57, 1-11. [CrossRef] [PubMed]

24. Preuß, M.; Nieuwenhuijsen, M.; Márquez, S.; Cirach, M.; Dadvand, P.; Triguero-Mas, M.; Gidlow, C.; Grazuleviciene, R.; Kruize, H.; Zijlema, W. Low childhood nature exposure is associated with worse mental health in adulthood. Int. J. Environ. Res. Public Health 2019, 16, 1809. [CrossRef]

25. Zijlema, W.; Ávila, I.; Triguero, M.; Gidlowd, C.; Maase, J.; Kruizef, H.; Andrusaityte, S.; Grazuleviciene, R.; Nieuwenhuijsen, M.J. Active commuting through natural environments is associated with better mental health: Results from the PHENOTYPE project. Environ. Int. 2018, 121, 721-727. [CrossRef] [PubMed]

26. Trøstrup, C.H.; Christiansen, A.B.; Stølen, K.S.; Nielsen, P.K.; Stelter, R. The effect of nature exposure on the mental health of patients: A systematic review. Qual. Life Res. 2019, 28, 1695. [CrossRef]

27. Butler, D.; Moseley, L.; Sunyata, A. Explain Pain; Noigroup Publications: Adelaide, Australia, 2016.

28. Bowler, D.E.; Buyung, L.M.; Knight, T.M.; Pullin, A.S. A systematic review of evidence for the added benefits to health of exposure to natural environments. BMC Public Health 2010, 10, 456. [CrossRef]

29. Bratman, G.N.; Hamilton, J.P.; Hahn, K.S.; Daily, G.C.; Gross, J.J. Nature experience reduces rumination and subgenual prefrontal cortex activation. Proc. Natl. Acad. Sci. USA 2015, 112, 8567-8572. [CrossRef]

30. Thompson, J.; Boddy, K.; Stein, K.; Whear, R.; Barton, J.; Depledge, M.H. Does participating in physical activity in outdoor natural environments have a greater effect on physical and mental wellbeing than physical activity indoors? A systematic review. Environ. Sci. Technol. 2011, 45, 1761-1772. [CrossRef]

31. Berman, M.G.; Kross, E.; Krpan, K.M.; Askren, M.K.; Burson, A.; Deldin, P.J.; Kaplan, S.; Sherdell, L.; Gotlib, I.H.; Jonides, J. Interacting with nature improves cognition and affect for individuals with depression. J. Affect. Disord. 2012, 140, 300-305. [CrossRef]

32. Luttenberger, K.; Stelzer, E.M.; Först, S.; Schopper, M.; Kornhuber, J.; Book, S. Indoor rock climbing (bouldering) as a new treatment for depression: Study design of a waitlist-controlled randomized group pilot study and the first results. BMC Psychiatry 2015, 15, 201. [CrossRef]

33. López-Pousa, S.; Bassets, G.; Monserrat, S.; Blanco, M.; Colomé, J.H.; Garre-Olmo, J. Sense of well-being in patients with fibromyalgia: Aerobic exercise program in a mature forest-A pilot study. Evid. Based Complement. Alternat. Med. 2015, 1-9. [CrossRef] [PubMed]

34. Chan, A.W.; Tetzlaff, J.M.; Altman, D.G.; Laupacis, A.; Gøtzsche, P.C.; Krleža-Jerić, K.; Hróbjartsson, A.; Mann, H.; Dickersin, K.; Berlin, J.A.; et al. SPIRIT 2013 statement: Defining standard protocol items for clinical trials. Ann. Intern. Med. 2013, 158, 200-207. [CrossRef] [PubMed]

35. Schulz, K.F.; Altman, D.G.; Moher, D. CONSORT 2010 Statement: Updated guidelines for reporting parallel group randomized trials. BMJ Med. 2010, 8, 18. [CrossRef] [PubMed] 
36. Ministerio de Sanidad, Política Social e Igualdad. Fibromialgia. Available online: https://www.mscbs.gob.es/ profesionales/prestacionesSanitarias/publicaciones/docs/fibromialgia.pdf (accessed on 18 January 2020).

37. Wolfe, F.; Clauw, D.J.; FitzCharles, M.; Goldenberg, D.L.; Häuser, W.; Katz, R.S.; Mease, P.; Russell, A.S.; Russell, I.J.; Winfield, J.B. Fibromyalgia criteria and severity scales for clinical and epidemiological studies: A modification of the ACR preliminary diagnostic criteria for fibromyalgia. J. Rheumatol. 2011, 38, 1113-1122. [CrossRef] [PubMed]

38. Segura-Jiménez, V.; Aparicio, V.A.; Álvarez-Gallardo, I.C.; Soriano-Maldonado, A.; Estévez-López, F.; Delgado-Fernández, M.; Carbonell-Baeza, A. Validation of the modified 2010 American College of Rheumatology diagnostic criteria for fibromyalgia in a Spanish population. Rheumatology 2014, 53, 1803-1811. [CrossRef]

39. Serrat, M.; Méndez-Ulrich, J.; Mateo-Canedo, C.; Jiménez-Titos, D.; Fontcuberta, A.; Sanz, A. Psychological Aspects of Fibromyalgia: Is There an Affective and Personality Profile of People Affected by Fibromyalgia? In Proceedings of the XII International Congress of the Spanish Society of Anxiety and Stress (SEAS), Barcelona, Spain, 8 September 2018.

40. Serrat, M.; Casimiro, J.; Jiménez, D.; Romeu, A.; Alonso, S.; Méndez-Ulrich, J.L.; Sanz, A. Panel of Experts for the Characterization of the Therapeutic Potential for the Psychological Intervention of Activities at Wilderness. In Proceedings of the III Congreso de Psicología Sanitaria y Salud Mental, Barcelona, Spain, 7-8 June 2018.

41. Sanabria-Mazo, J.P.; Serrat, M.; Mateo-Canedo, C.; Feliu-Solera, A.; Almirall, M.; Méndez-Ulricha, J.L.; Luciano, J.V.; Sanz, A. Proof of concept of a multicomponent intervention for fibromyalgia based on physical activity, psychological support, and exposure to nature (NAT-FM). J. Comp. Eff. Res. Under review.

42. May, M.; Junghaenel, D.U.; Ono, M.; Stone, A.A.; Schneider, S. Ecological momentary assessment methodology in chronic pain research: A systematic review. J. Pain 2018, 19, 699-716. [CrossRef]

43. First, M.B.; Spitzer, R.L.; Gibbon, M. Structured Clinical Interview for DSM-IV Axis I Disorders, Clinician Version; American Psychiatric Association: Washington, DC, USA, 1997.

44. Luciano, J.V.; Aguado, J.; Serrano, A.; Calandre, E.P.; Rodriguez-Lopez, C.M. Dimensionality, reliability, and validity of the Revised Fibromyalgia Impact Questionnaire in two Spanish samples. Arthritis Care Res. 2013, 65, 1682-1689. [CrossRef]

45. López, I.; Hervás, G.; Vázquez, C. Adaptación de la "Escala de afecto positivo y negativo" (PANAS) en una muestra general española. Behav. Psychol. 2015, 23, 529-548.

46. Feliu-Soler, A.; Reche, E.; Borràs, X.; Pérez-Aranda, A.; Andrés-Rodríguez, L.; Peñarrubia-María, M.; Navarro-Gil, M.; García-Campayo, J.; Bellón, J.; Luciano, J.V. Psychometric properties of the Cognitive Emotion Regulation Questionnaire (CERQ) in patients with fibromyalgia syndrome. Front. Psychol. 2017, 8 , 2075. [CrossRef]

47. Fernández, J.; Doval, E.; Blasco, T.; Sanz-Ruiz, A.; Álvarez, M. Validación de la Escala de Competencia Personal de Wallston: Implicaciones para el estudio del estrés. Ans. Est. 1998, 4, 31-41.

48. Vázquez, A.J.; Vázquez, R. Escala de Autoestima de Rosenberg: Fiabilidad y validez en población clínica española. Apunt. Psicol. 2004, 22, 247-255.

49. García, J.; Rodero, B.; Alda, M.; Sobradiel, N.; Montero, J.; Moreno, S. Validación de la versión española de la escala de la catastrofización ante el dolor (Pain Catastrophizing Scale) en la fibromialgia. Med. Clín. 2008, 131, 487-492. [CrossRef] [PubMed]

50. Pedrero, E.J.; Ruiz, J.M.; Lozoya, P.; Rojo-Mota, G.; Llanero-Luque, M.; Puerta-García, C. La “Escala de Estrés Percibido": Estudio psicométrico sin restricciones en población no clínica y adictos a sustancias en tratamiento. Behav. Psychol. 2015, 23, 305-324.

51. Vallejo, M.A.; Rivera, J.; Esteve, J.; Rodríguez-Muñoza, M.F.; Grupo ICAF. Uso del cuestionario Hospital Anxiety and Depression Scale (HADS) para evaluar la ansiedad y la depresión en pacientes con fibromialgia. Rev. Psiquiatr. Salud. 2012, 5, 107-114. [CrossRef]

52. Luciano, J.V.; Barranda, J.R.; Aguado, J.; Osma, J.; García-Campayo, J. Bifactor analysis and construct validity of the HADS: A cross-sectional and longitudinal study in fibromyalgia patients. Psychol. Assess. 2014, 26, 395-406. [CrossRef] 
53. Rodero, B.; Pereira, J.B.; Pérez, M.C.; Casanueva, B.; Serrano-Blanco, A.; Rodrigues-Da-Cunha-Ribeiro, M.J.; Luciano, J.V.; Garcia-Campayo, J. Validation of a Spanish version of the Psychological Inflexibility in Pain Scale (PIPS) and an evaluation of its relation with acceptance of pain and mindfulness in sample of persons with fibromyalgia. Health Qual. Life Outcomes 2013, 11, 62. [CrossRef]

54. Gómez, L.; López, A.E.; Ruiz, G.T. Psychometric properties of the Spanish version of the Tampa Scale for Kinesiophobia (TSK). J. Pain 2011, 12, 425-435. [CrossRef]

55. Vilagut, G.; Ferrer, M.; Rajmil, L.; Rebollo, P.; Permanyer-Miralda, G.; Quintana, J.M.; Santed, R.; Valderas, J.M.; Ribera, A.; Domingo-Salvany, A.; et al. The Spanish version of the Short Form 36 Health Survey: A decade of experience and new developments. Gac. Sanit. 2005, 19, 135-150. [CrossRef]

56. Oja, P.; Laukkanen, R.; Pasanen, M.; Tyry, T.; Vuori, I. A 2-km walking test for assessing the cardiorespiratory fitness of healthy adults. Int. J. Sports Med. 1991, 12, 356-362. [CrossRef]

57. Guy, W. ECDEU Assessment Manual for Psychopharmacology; US Department of Health, Education, and Welfare Public Health Service Alcohol, Drug Abuse, and Mental Health Administration: Rockville, MD, USA, 1976.

58. Scott, W.; McCracken, L.M. Patient's impression of change following treatment for chronic pain: Global Specific, a single dimension, or many? J. Pain 2015, 16, 518-526. [CrossRef]

59. Bradley, M.M.; Lang, P.J. Measuring emotion: The Self-Assessment manikin and the semantic differential. J. Behav. Ther. Exp. Psychiatry 1994, 25, 49-59. [CrossRef]

60. Luciano, J.V.; Guallar, J.A.; Aguado, J.; López-Del-Hoyo, Y.; Olivan, B.; Magallón, R.; Alda, M.; Serrano-Blanco, A.; Gili, M.; Garcia-Campayo, J. Effectiveness of group acceptance and commitment therapy for fibromyalgia: A 6-month randomized controlled trial (EFFIGACT study). Pain 2014, 155, 693-702. [CrossRef] [PubMed]

61. Thissen, D.; Steinberg, L.; Kuan, D. Quick and Easy Implementation of the Benjamini Hochberg Procedure for Controlling the False Positive Rate in Multiple Comparisons. J. Edu. Behav. Stat. Spring 2002, 27, 77-83. [CrossRef]

62. Romeu, A.; Alonso, S.; Serrat, M.; Mateo-Canedo, C.; Sanabria-Mazo, J.P.; Méndez-Ulrich, J.L.; Sanz, A. MOTI-NAT-FM: Determinants of Adherence to Therapies Based on Physical Activity in Nature for the Treatment of Fibromyalgia. In Proceedings of the IV Congreso de Psicología Sanitaria y Salud Mental, Barcelona, Spain, 6 June 2019.

(C) 2020 by the authors. Licensee MDPI, Basel, Switzerland. This article is an open access article distributed under the terms and conditions of the Creative Commons Attribution (CC BY) license (http://creativecommons.org/licenses/by/4.0/). 